\title{
Nonlinear Compressive Sensing for Distorted Measurements and Application to Improving Efficiency of Power Amplifiers
}

\section{Citation}

Chen, Hsieh-Chung, H.T. Kung, and Marcus Comiter. 2017. Nonlinear Compressive Sensing for Distorted Measurements and Application to Improving Efficiency of Power Amplifier. In 2017 IEEE International Conference on Communications (ICC), 1-7. Paris: IEEE.

\section{Permanent link}

http://nrs.harvard.edu/urn-3:HUL.InstRepos:40996254

\section{Terms of Use}

This article was downloaded from Harvard University's DASH repository, and is made available under the terms and conditions applicable to Open Access Policy Articles, as set forth at http:// nrs.harvard.edu/urn-3:HUL.InstRepos:dash.current.terms-of-use\#OAP

\section{Share Your Story}

The Harvard community has made this article openly available. Please share how this access benefits you. Submit a story.

Accessibility 


\title{
Nonlinear Compressive Sensing for Distorted Measurements and Application to Improving Efficiency of Power Amplifiers
}

\author{
Hsieh-Chung Chen, H.T. Kung, Marcus Comiter \\ Harvard University
}

\begin{abstract}
Compressive sensing, which enables signal recovery from fewer samples than traditional sampling theory dictates, assumes that the sampling process is linear. However, this linearity assumption may not hold in the analog domain without significant trade-offs, such as power amplifiers sacrificing substantial power efficiency in exchange for producing linear outputs. Since compressive sensing is most impactful when implemented in the analog domain, it is of interest to integrate the nonlinearity in compressive measurements into the signal recovery process such that nonlinear effects can be mitigated. As such, in this paper, we describe a nonlinear compressive sensing formulation and associated signal recovery algorithms, providing both compression and improved efficiency of a power amplifier simultaneously with one procedure. We present evaluations of the proposed framework using both measurements from real power amplifiers and simulations.
\end{abstract}

Index Terms-Compressive sensing, sparse coding, efficiency of power amplifier, nonlinear measurements, sparse signal recovery

\section{INTRODUCTION}

Compressive sensing (CS) enables signal recovery from fewer samples than traditional sampling theory dictates. By introducing a sparsity constraint during signal recovery, a sparse signal can be recovered from just a relatively few measurements [1], [2]. The recovery process involves solving a mathematical optimization problem.

Under a scenario in which the sensing device is relatively constrained in power, compressive sensing can be useful in reducing both the sensing and compression cost. For example, consider the scenario shown in Figure 1, in which one wants to sense signal using a mobile device and then send it to the cloud. Alleviating the burden of signal sensing with linear compressive sampling from the relatively resource-constrained mobile device at the cost of additional computation effort at the resource-rich cloud, which is equipped to handle a computationally more expensive decoding process, would be a desired tradeoff in this case.

While compressive sensing assumes that the sampling process is linear, this is often difficult to achieve in the analog domain without significant trade-offs. For example, a power amplifier (PA) tends to sacrifice substantial power efficiency in exchange for producing linear outputs [3]. Since compressive sensing is most impactful when implemented in the analog domain, it is desirable to alleviate the effect of nonlinearity

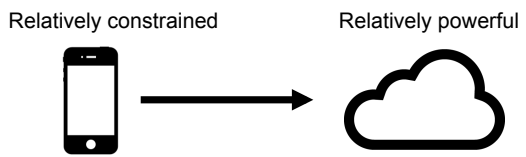

Fig. 1. Compressive sensing shifts some burden of signal acquisition from the front end to back end. This tradeoff is favorable when we have powerconstrained sensing devices paired with relatively powerful backend.

in sampling by integrating nonlinearity considerations into the decoding process of signal recovery, as doing so would allow for greater power amplifier efficiency by reducing linearity requirements.

In this paper, we present a nonlinear compressive sensing (NLCS) formulation that directly models the nonlinear distortion to compressive measurements in the decoding process, and the accelerated proximal gradient descent method that can efficiently solve the optimization problem in our new formulation. By handling the nonlinear distortion with NLCS, our proposed methodology benefits systems in which front end devices are power-constrained. Specifically, by lowering the linearity requirements for analog devices, our approach could allow amplifiers to operate at higher power efficiency, which is currently infeasible due to significant distortion. Our approach still has the benefit of conventional compressive sensing, that is, the use of simple linear encoding to compress data for reduced transmission cost.

The proposed NLCS methodology is applicable to nonlinear distortions that are smooth and differentiable. This is a property that generally holds for many analog devices such as amplifiers. The optimization process associated with NLCS can handle unknown distortion functions by estimating said functions along with signal recovery. To validate the proposed methodology, we present both simulated results with nonlinear models that are used in the amplifier industry, as well as evaluations using measurements from real power amplifiers.

We make the following key contributions in this paper:

1) We provide an analysis that proves signal recoverability for a practical nonlinear compressive sensing model.

2) We present a signal recovery algorithm for solving the optimization problem associated with nonlinear compressive sensing, and an extension that estimates the distortion 
function during signal recovery.

3) We contribute the first known empirical results showing the power efficiency gain via nonlinear compressive sensing using data from a real power amplifier.

In Section II, we discuss relevant background. In Section III, we introduce the NLCS framework with extensions to handle unknown distortions. In Section IV, we provide analysis to show that information required for signal recovery is preserved in the nonlinear sensing setting. In Section V, we present empirical results from simulation and real hardware.

\section{BACKGROUND}

\section{A. Compressive Sensing}

Compressive sensing is a framework in which signals are sampled through a linear projection $\Phi \in \mathbb{R}^{m \times n}$, where $m<<$ $n$. We assume that all signals $x \in X$ can be sparsely expressed as $x=\Psi z$, where $\Psi \in \mathbb{R}^{n \times t}$ is a (overcomplete) basis and $z$ is a sparse vector. The basis $\Psi$ is designed or trained, and we assume it is given. The measurement $y$ is defined as follows:

$$
y=\Phi x+\epsilon=\Phi \Psi z+\epsilon
$$

where $\epsilon$ denotes the noise in measurements.

Given $y$, one can recover $x$ indirectly by first finding a sparse vector $z$ that explains the measurements. There are several formulations for computing $z$ with different performance guarantees, but the general solution is

$$
z^{*}=\underset{z}{\arg \min } g(z) \text { subject to }\|y-\Phi \Psi z\|_{2} \leq\|\epsilon\|_{2}
$$

where $g$ is an objective function that promotes sparsity (e.g., $\left.g(z)=\|z\|_{1}\right)$. Once $z^{*}$ is acquired, one can obtain an estimation of $x$ via $x^{*}=\Psi z^{*}$. The error of such recovery depends on the properties of $\Phi \Psi$, the sparsity of $z$, and the choice of the regularization function $g^{1}$.

Definition 1 (restricted isometry property). A matrix A satisfies the restricted isometry property (RIP) with respect to parameters $\delta, k$ if the following is true for all $k$ sparse vectors $x \in \mathbb{R}^{n}$ :

$$
(1-\delta)\|x\|_{2}^{2} \leq\|A x\|_{2}^{2} \leq(1+\delta)\|x\|_{2}^{2}
$$

If the real signal $z$ is approximately $k$ sparse and $\Phi \Psi$ satisfies $\operatorname{RIP}(\sqrt{2}-1,2 k)$ (see Definition 1), then the solution found by (2) using $g(z)=\|z\|_{1}$ would satisfy

$$
\left\|z^{*}-z\right\|_{2} \leq c_{0} \frac{\left\|z-z_{k}\right\|_{1}}{\sqrt{k}}+c_{1} \epsilon
$$

where $z_{k}$ is a truncated version of $z$ with only the largest $k$ coefficients.

\footnotetext{
${ }^{1}$ In the case when a nonconvex $g$ is used, the performance would also depend on the optimization algorithm for solving (2).
}

\section{B. Related Work on Nonlinear Measurements}

There has been a line of research by Jacques et al. that specifically addresses the quantization effect of analog-digital converters (ADC) [4]. In their formulation, the measurements are quantized into discrete numbers (binary in the extreme case). That is, they assume $y^{\prime}=f(\Phi x)$ where $f$ is a quantization function $(f(x)=\operatorname{sign}(x)$ in the extreme case). Their work models the quantization effect of ADCs, and involves a solver specialized to the discrete nature of their problem. However, this discrete model does not apply to the continuous distortion that we consider in this paper.

Boufounos proposes a model to handle order-preserving distortions [5]. This line of work is similar to Jacques' approach in spirit, in that it makes weak assumptions about the measurements. Their reconstruction only relies on order statistics and does not try to recover information about signal magnitude.

On a different front, Blumensath focuses on the problem of having $y^{\prime}=\Upsilon(x)$, where $\Upsilon(\cdot)$ is a general sensing function instead of a sensing matrix, and shows that $x$ can be recovered if $\Upsilon$ satisfies some RIP-like properties. Specifically, it is assumed that $\Upsilon(x)$ is a function that has a proper linear approximation. If the Jacobian of $\Upsilon(x)$ satisfies the restricted isometry property, then the reconstruction error will be bounded [6]. However, there is currently no easy way to verify if a given $\Upsilon$ satisfies such assumption. As we will describe later, a more specific type of nonlinear sensing process can easily be shown to preserve information necessary for signal recovery.

Finally, Thrampoulidis and Abbasi recently developed a precise performance characterization of LASSO with nonlinear measurements, and showed that LASSO is a good estimator under the presence of nonlinear distortion [7]. Specifically, the authors treat $y^{\prime}=f(\Phi x)$ as $y^{\prime}=\Phi x+\epsilon$, and show that the solution in (2) would still yield an estimation with bounded error. However, this approach does not take the knowledge of $f$ into account. In this paper, we exploit known knowledge about $f$ (e.g., the Rapp function can model $f$ ) to achieve lower reconstruction error.

\section{Distortion: Amplifier Characteristics}

We focus on the power amplifier as an example of analog device exhibiting nonlinearity in compressive measurements, noting that it is one of the prominent bottlenecks in wireless communication. In a RF transmitter operating in millimeter frequency bands, its power amplifier is the main consumer of power, and yet it normally only achieves a low efficiency, which can be as low as 10 percent. This creates significant problems both from its high power consumption and its requisite cooling, as the dissipated power is transformed into heat.

However, there is a tradeoff between the power efficiency of an amplifier and the linearity of a power amplifier's output [8]. If the requirement for power amplifier output linearity is lowered, the amplifier can in fact operate at much higher efficiency. See Figure 2 for an example of amplifier nonlinearity. 


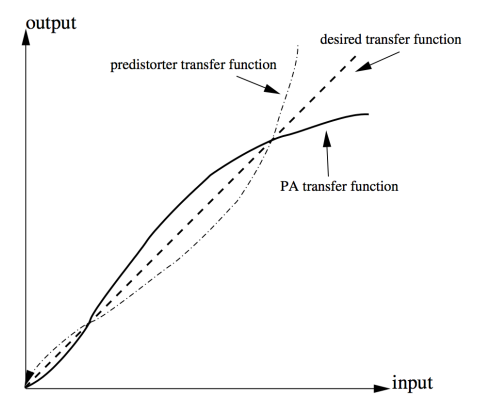

Fig. 2. Illustration of amplifier distortion and the predistortion technique.

For an amplifier to operate linearly, the input signal must fall into the linear region. When there is a large dynamic range of inputs, as is the case with compressive sensing ${ }^{2}$, amplifiers with larger linear regions are needed. However, this larger linear region comes at a cost of lower efficiency.

One of the techniques used in practice to mitigate this problem is predistortion, as illustrated in Figure 2. The principle idea of predistortion methods is to measure the distortion function beforehand, and apply an inverse of that distortion function prior to amplifiers so that the effect cancels out [9]. While both predistortion and NLCS addresses the same problem of nonlinear distortion, they do so in different manners. Predistortion addresses the nonlinearity at the amplifier during signal acquisition, while NLCS attempts to mitigate the nonlinearity after signal acquisition. As such, NLCS can be applied regardless of if predistortion is also applied, making our method orthogonal and complementary to predistortion.

\section{Nonlinear Compressive SEnsing}

We now detail nonlinear compressive sensing (NLCS). First, we describe the signal acquisition process with nonlinear distortion explicitly modeled in the formulation. We then present the modified compressive sensing problem and suggest an optimization algorithm for solving it. Finally, we present an algorithm for estimating both the distortion function and the original sparse signal simultaneously.

\section{A. Signal Acquisition}

In this paper, we assume that the sensed signal $y^{\prime}$ can be represented as distorted measurements

$$
\begin{aligned}
y^{\prime} & =f(\Phi x)+\epsilon \\
& =f(\Phi \Psi z)+\epsilon
\end{aligned}
$$

through some nonlinear distortion function $f$. We assume that the nonlinearity $f$ is of a single variate (i.e., the effect of $f$ on each number in the vector is independent, and is therefore the same as applying a univariate $f$ on all the inputs). However, this could be extended to a multivariate nonlinearity functions. As in standard compressive sensing, $\Phi$ is the sensing matrix,

\footnotetext{
${ }^{2}$ Compressive sensing measurements normally have a large dynamic range because each measurement is a random sum of a large number of variables.
}

and we assume $x=\Psi z$, for some sparsifying basis $\Psi$. We further assume that $f$ is differentiable.

There are many different models in the literature for power amplifier distortion. We will use the Rapp model in our experiments, as it is one of the most widely used models [10], where $f$ is defined as:

$$
f(x) \equiv \frac{x}{\left.\left(1+\left(\frac{x}{\alpha}\right)^{2 \beta}\right)\right)^{\frac{1}{2 \beta}}}
$$

with parameters $\alpha$ and $\beta$ controlling the shape of the function. One common attribute of all the models is that the distortion is some form of saturation, as illustrated in Figure 2 [11]. Note that while results in this paper are presented on the Rapp model, we have observed similar results for other models as well.

\section{B. Signal Recovery}

Given the distorted measurement $y^{\prime}$, the signal can be recovered as follows:

$$
z^{*}=\underset{z}{\arg \min } g(z) \text { subject to }\|y-f(\Phi \Psi z)\|_{2} \leq\|\epsilon\|_{2}
$$

Alternatively, one can use the following regularized form, which is equivalent to (8) for some $\lambda$. This can be interpreted as balancing the fitness $Q(z)=\|y-f(\Phi \Psi z)\|_{2}^{2}$ and sparsity constraint $g(z)$ of $z$ by tuning the regularization parameter $\lambda$ :

$$
z^{*}=\underset{z}{\arg \min } Q(z)+\lambda g(z)
$$

Assuming that $f$ is differentiable, we can find a solution using accelerated proximal gradient descent (FISTA) [12]. We use a similar treatment to separate the smooth part $\|y-f(\Phi \Psi z)\|_{2}^{2}$ and potentially non-smooth $\lambda g(z)$ in the cost function of (9), and define the gradient step as:

$$
z^{k}=\operatorname{prox}_{\eta \lambda g}\left(z^{k-1}+\eta \partial Q(z)\right)
$$

where $\eta$ is the stepsize, and $\operatorname{prox}_{h}(w)$ denotes the proximal operator associated with the regularization function:

$$
\operatorname{prox}_{h}(w)=\underset{v}{\arg \min } \frac{1}{2}\|v-w\|_{2}^{2}+h(v)
$$

When $g$ is the $\ell_{0}$ or $\ell_{1}$ norm, the corresponding proximal operator is the hard-threshold or soft-threshold function, respectively.

The full algorithm with acceleration and restart is shown in Algorithm 1. When the objective is locally convex, this algorithm has quadratic convergence rate. That is,

$$
F\left(z^{k}\right)-F(z) \leq \frac{c\left\|z^{0}-z\right\|_{2}^{2}}{(k+1)^{2}}
$$

for $k$ th iterate $z^{k}$, where $F$ is the objective as defined in Algorithm 1, and $c$ is a constant related to stepsize $\eta$ and smoothness of $Q(z)$. The algorithm terminates when $\left\|z^{k}-z^{k+1}\right\|$ becomes sufficiently small ${ }^{3}$. Note that there are other optimization techniques that one can adopt if efficiency is a primary concern (e.g., [13], [14]).

\footnotetext{
${ }^{3}$ The threshold is usually selected so that every step makes meaningful progress, which is application dependent.
} 


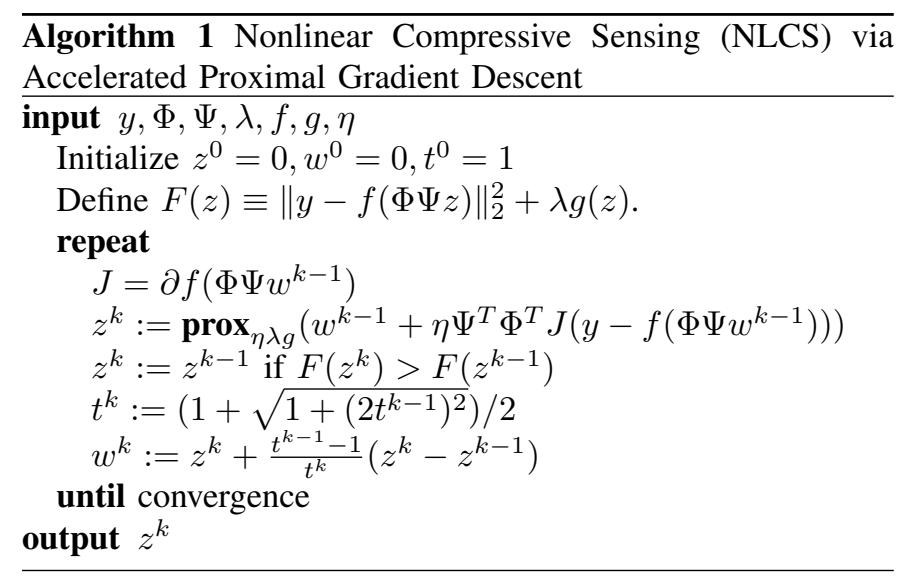

\section{Distortion Estimation}

In this section we assume that the distortion function $f$ is controlled by some parameter $\alpha \in \mathbb{R}^{\beta}$, and that the measurements are $y^{\prime}=f_{\alpha}(\Phi \Psi z)$. Note that even in the case where the parameters of the distortion function are unknown, it is still possible to estimate these parameters jointly with the signal. In a fashion similar to the treatment of (9), the parameters are treated as additional unknown variables, and the joint recovery is done through the following optimization:

$$
z^{*}, \alpha^{*}=\underset{z, \alpha}{\arg \min }\left\|y-f_{\alpha}(\Phi \Psi z)\right\|_{2}^{2}+\lambda g(z)
$$

To solve (13), we use block coordinate descent [14] that alternates between $z$ and $\alpha$. The details are described in Algorithm 2.

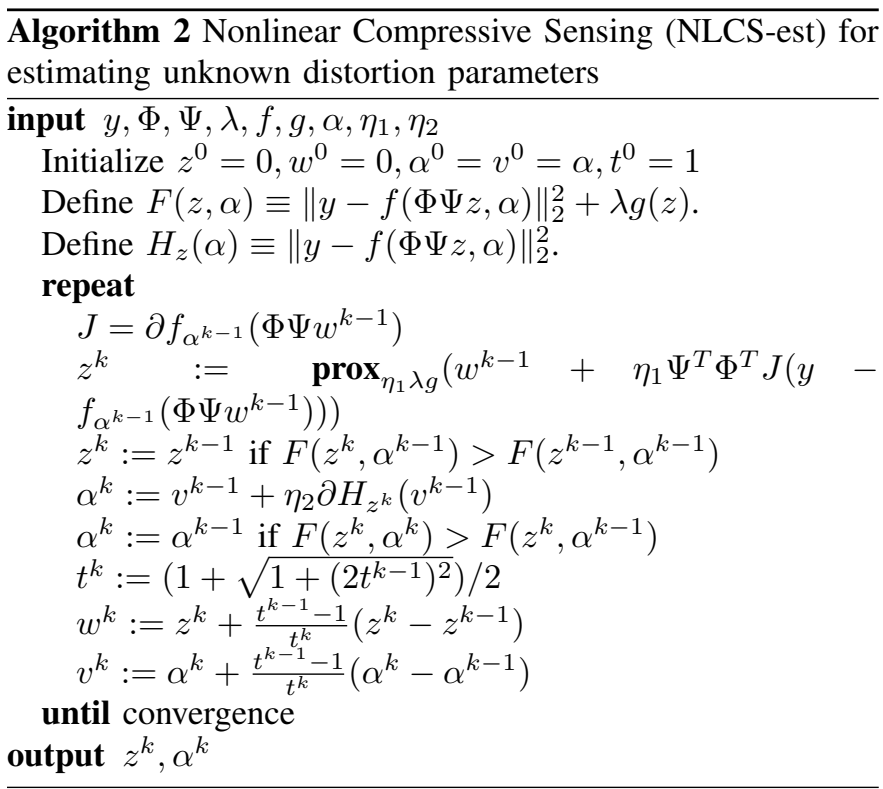

\section{ANALYSIS}

In this section, we consider distortion functions that do not alter the signal significantly. In most applications, the distortion is in the form of saturation or compression, both of which preserve relative distances rather well. We first concretely define the properties of these distortion functions.

Definition 2 (limited distortion). A function $f: \mathbb{R}^{m} \rightarrow \mathbb{R}^{m}$ is $\alpha$-isometry if the following holds for any $x, y \in \mathbb{R}^{m}$ :

$$
(1-\alpha)\|x-y\|_{2}^{2} \leq\|f(x)-f(y)\|_{2}^{2} \leq(1+\alpha)\|x-y\|_{2}^{2}
$$

One of the key ideas of compressive sensing is that information in the original signal can be preserved through a low dimensional projection. We now show that this still holds when the measurements are distorted by $\alpha$-isometry functions.

Theorem 1. Let $f$ be an $\alpha$-isometry function with $\alpha<1$. Let $(\Phi \Psi)$ be a matrix that satisfies RIP with parameters $\delta, 2 k$. Let $z$ be a vector such that $|z|_{0} \leq k$, and let $y=f(\Phi \Psi z)$ be the compression of $z$. Let

$$
z^{*}=\underset{z}{\arg \min }\|z\|_{0} \quad \text { subject to } \quad y=f(\Phi \Psi z)
$$

be the reconstructed vector. Then, $z^{*}=z$.

Proof. Since $f(\Phi \Psi z)-f\left(\Phi \Psi z^{*}\right)=0$, by Definition 2 we can conclude that $\Phi \Psi\left(z-z^{*}\right)=0$. Let $\hat{z}=z^{*}-z$, then we have $\|\hat{z}\|_{0} \leq 2 k$ (since $\|z\|_{0},\left\|z^{*}\right\|_{0} \leq k$ ). Since $\Phi \Psi$ satisfies RIP with parameters $\delta, 2 k$ and $\|\hat{z}\|_{0} \leq 2 k$, it must be that $\hat{z}=0$ in order to satisfy $\Phi \Psi(\hat{z})=0$.

While Theorem 1 guarantees that the signal can be recovered with some reconstruction scheme, the objective is combinatorial and could be computationally expensive to solve. To address this, we leverage one of the other key results in standard compressive sensing, namely that minimizing the $\ell_{1}$ norm can be a surrogate for minimizing the $\ell_{0}$ norm, allowing efficient convex programming algorithms to be used. The same principle also applies in our nonlinear version.

Theorem 2. Let $f$ be a $\alpha$-isometry function with $\alpha<1$. Let $(\Phi \Psi)$ be a matrix that satisfies RIP with parameters $\delta, 2 k$. Let $y=f(\Phi \Psi z)$ be the compression of $z$. Let

$$
z^{*}=\underset{z}{\arg \min }\|z\|_{1} \quad \text { subject to } \quad y=f(\Phi \Psi z)
$$

be the reconstructed vector. Then,

$$
\left\|z^{*}-z\right\|_{2} \leq c_{\delta, \alpha} \frac{\left\|z-z_{k}\right\|_{1}}{\sqrt{k}}
$$

where $c_{\delta, \alpha}$ is a constant, and $z_{k}$ is a truncated version of $z$ formed by keeping only the largest $k$ coefficients, i.e.,

$$
z_{k}=\underset{v:\|v\|_{0} \leq k}{\arg \min }\|z-v\|_{2}
$$

One caveat is that the constraint in (16) becomes non-convex in nonlinear compressive sensing. Therefore, the NLCS solver may not necessarily converge as well as in standard CS. While the theoretical convergence guarantee is still under study, we have observed promising reconstruction rates empirically. 
Although the NLCS objective is not convex, it will still converge to the global optimal if the distortion function is element-wise monotonic. This is reflected in the NLCS loss function shown in Figure 3(b) based on the Rapp distortion model, which is convex in the neighborhood around 0 and has global convergence. As such, the algorithm can still converge efficiently as soon as it reaches the regions around the solution.

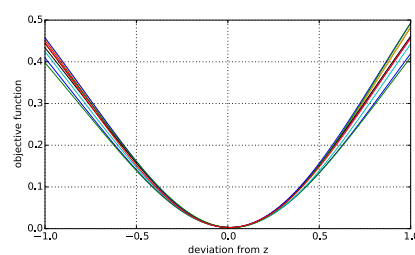

(a) CS loss function

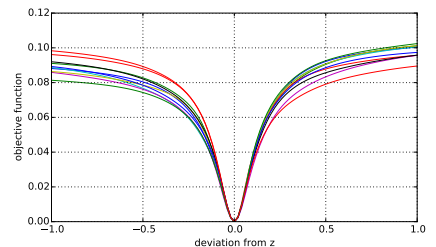

(b) NLCS loss function

Fig. 3. The loss function of NLCS (right) becomes nonconvex due to the nonlinear distortion function, as compared to the convex loss function of standard CS (left), where each line is the loss function for a different signal under the Rapp model with a different $\alpha$ value. Each line is drawn by moving away from the ground truth $z$ with respect to some random direction.

\section{EMPIRICAL RESULTS}

In this section, we present results validating the proposed methodology. We first describe the data generation process and experimental setup. Next, we present results from simulations, in which we show the signal recovery quality of NLCS under varying conditions. Following this, we present results from applying NLCS to measurements obtained from a real world power amplifier, and demonstrate the efficiency gains realized by tolerating nonlinear distortions.

\section{A. Data Generation and Experimental Setup}

Before presenting results, we first detail the data generation process used for both the simulation and real power amplifier results. All signals are generated randomly as follows. First, a random dictionary $\Psi$ is generated, where each column in $\Psi$ is made up of random draws from a Gaussian distribution with mean 0 and variance 1 . Next, a sparse vector $z$ of length equal to the number of columns in $\Psi$ is formed by randomly choosing $k$ indices, drawing a value for each of the $k$ indices from a Gaussian distribution with mean 0 and variance 1 , and setting the remaining entries of $z$ to 0 . Finally, the following two quantities are computed: $X=\Psi z$ and $Y=\Phi \Psi z$, where matrix $\Phi$ is created by randomly drawing each entry from a Gaussian distribution with mean 0 and variance $1 . X$ and $Y$ are then passed through the noise model (simulation results) or power amplifier (real PA results) to produce the distorted signal $f(X)$ and $f(Y)$ from which the original signal is recovered.

In recovering the signal using the formulation shown in equation 9 , the value of $\lambda$ is found via a screening process. While both $\alpha$ and $\beta$ can be recovered via NCLS, we focus on the effects of changing $\alpha$ for presentation simplicity.

For simulation results, reconstruction error is measured in terms of the $\ell_{2}$ norm between the reconstructed signal and the known ground truth signal. For real PA results, error is measured in terms of SNR, where SNR is calculated as the ratio of the power of the reconstructed signal to the power of the ground-truth original signal, which is known as it is generated. Finally, when comparing NLCS against CS, the CS method treats the nonlinearity as noise, and these "noisy" measurements are used in signal recovery.

\section{B. Simulations}

In this section, we consider several different distortion functions and show the reconstruction quality with and without nonlinearity in the compressive sensing formulation.

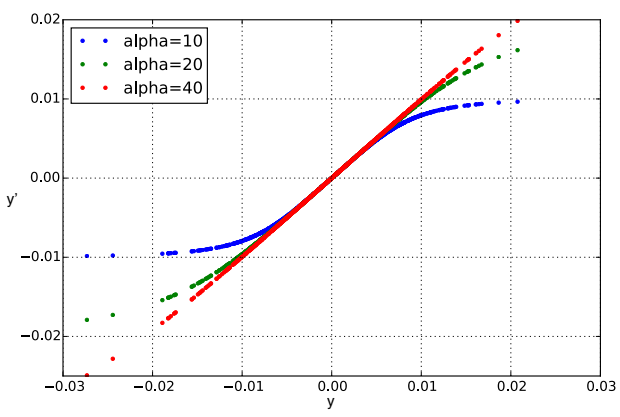

Fig. 4. Distortion function with different parameters. Each curve represents distortion based on the Rapp model with a different parameter $\alpha$.

We now examine the reconstruction quality under both traditional CS and NLCS. Specifically, we compare the two methods for distortion functions under different settings of $\alpha$ between 10 and 40, with the resulting distortion functions shown in Figure 4. The simulated results are shown in Figure 5. We find that the reconstruction quality is significantly better when nonlinearity is modeled directly, as in NLCS.

Additionally, we present results with an incorrect initial estimation provided to NLCS (which we denote by NLCSest), where NLCS-est is initiated with $\hat{\alpha}=\alpha+\epsilon$ where $\epsilon$ is drawn from a zero-mean unit-norm Gaussian distribution. We find that even in this case, in which the initial estimation is inaccurate, the signal can again be more successfully recovered via NLCS-est than it can be via traditional CS. However, we find that for NLCS-est, the variance of the reconstruction error increases as the distortion of the signal increases. This is due to the fact that the added distortion compounds the deleterious effect of inaccurately setting $\alpha$ that is otherwise largely insignificant at smaller levels of distortion.

The recovery quality over different compression rates is shown in Figure 6 for CS, NLCS, and NLCS-est. For these results, we set the distortion parameter $\alpha$ to 20 . Under this setting, the initial distortion parameter $\alpha$ for NLCS-est is set to be 10 , which deviates from the real curve as shown in Figure 4. Despite the inaccurate initial setting, NLCS-est is still able to deliver reconstruction results comparable to NLCS with a known distortion parameter $\alpha$, both of which again largely outperform traditional CS.

We find that these gains hold even as the initial estimate diverges further from the true value. This is shown in Figure 7, in which we set the true distortion parameter $\alpha$ at 20, yet use varying initial estimates for NLCS-est. Note that NLCS-est 


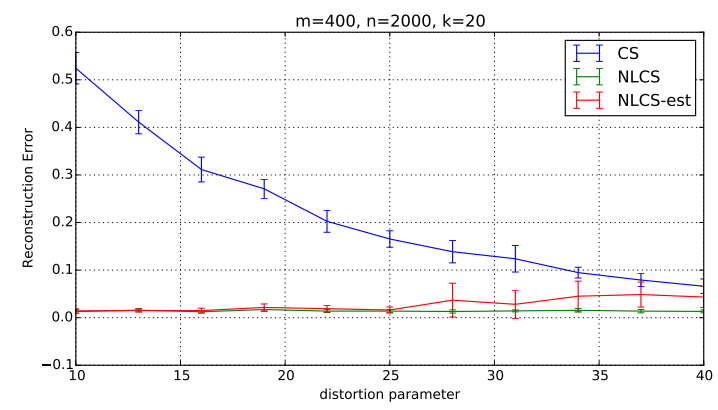

Fig. 5. Reconstruction error in $\ell_{2}$ norm of NLCS in comparison to standard compressive sensing. In this experiment, NLCS-est is initiated with $\hat{\alpha}=\alpha+\epsilon$ where $\epsilon$ is drawn from a zero-mean unit-norm Gaussian distribution.

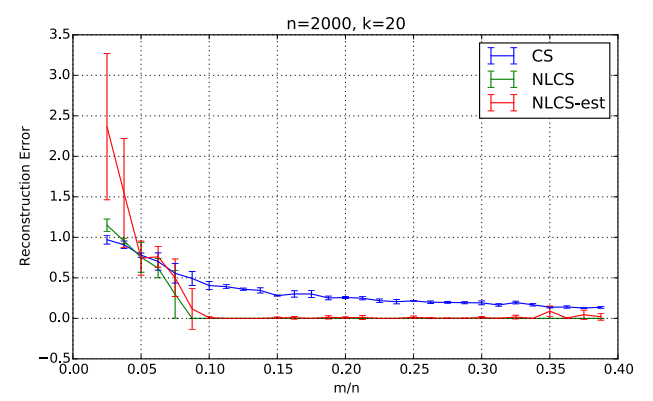

Fig. 6. Reconstruction error in $\ell_{2}$ norm at different compression rates. When the parameter of the distortion function is unknown, nonlinear CS can be used to estimate the distortion parameter jointly with the signal at the expense of slightly more measurement samples. (In this case, NLCS-est requires $10 \%$ measurements to achieve near-exact recovery, while NLCS only requires $8 \%$ ).

is able to overcome both overestimation and underestimation of the distortion nonlinearity. However, we find that the variance of the reconstruction error increases as the error in the estimated value of $\alpha$ increases. While this shows that the accuracy of the estimation does in fact impact performance, we note that even with an inaccurate estimate, the reconstruction error and its variance is still significantly lower than that of the CS method

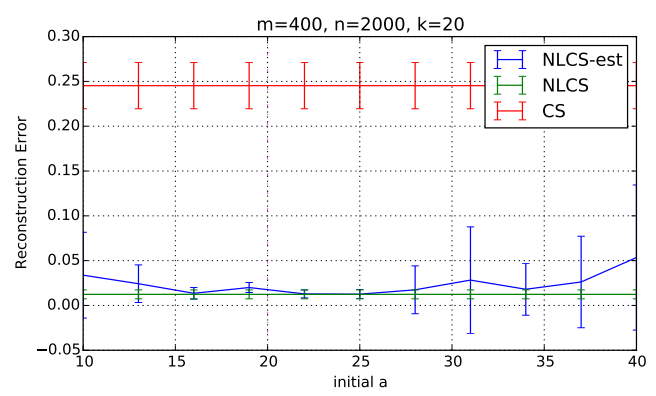

Fig. 7. NLCS-est can estimate the distortion parameter $\alpha$ jointly with the original signal. It reliably reconstructs the original signal even when the initial estimation of the distortion parameter $\alpha$ is inaccurate, where reconstruction error is in terms of $\ell_{2}$ error.

\section{Real PA Measurements}

We additionally present results on real power amplifier (PA) measurements. For these experiments, we use the MGA-43003
PA for $1.805-1.88 \mathrm{GHz}$ made by Avago Technologies [15]. This device is designed for small cell wireless communication in the $1.805-1.88 \mathrm{GHz}$ band. The tradeoff between power efficiency and linearity for this device is shown in Figure 9.

We operate the device at varying efficiency levels in order to test the signal recovery quality using CS and NLCS. At low efficiency (i.e., when the amplifiers is operating linearly), we provide input within the linear region of the device specification. In order to achieve higher efficiency, we increase the range of input samples so that some fall outside of the linear region in which the output would be distorted. We then try to reconstruct the original signal from the possibly distorted compressive measurements in order to show that the original signals can be recovered even when amplifiers do not respond linearly with respect to the large input range.

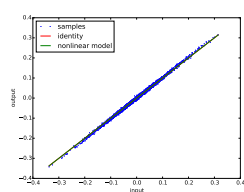

(a) $9 \%$

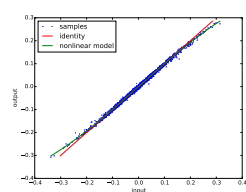

(b) $16 \%$

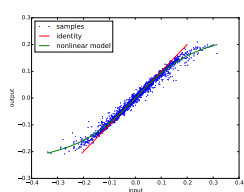

(c) $24 \%$
Fig. 8. Signal distortion measured at different PA power efficiency levels. The Rapp model is used to approximate the distortion nonlinearity. Note that at higher power efficiency, the thermal noise is also increased.

Figure 8 shows the distortion observed at different efficiency levels and a corresponding Rapp model. In the context of power amplifiers, power efficiency (or power-added efficiency, to be precise) is defined by:

$$
\mathrm{PAE}=\left(\left[P_{O U T}\right]-\left[P_{I N}\right]\right) /\left[P_{D C}\right]
$$

As described in Section II-C, the outputs would saturate when the PA becomes nonlinear. Note that in addition to nonlinearity, the thermal noise is also higher when the PA is operated at higher efficiency, which adds to the difficulty of signal reconstruction. We use NLCS-est to estimate the $\alpha$ parameter in the Rapp model. Based on the observations from our simulations, we set the initial value of the distortion parameter at 40 , which roughly corresponds to a linear $f$.

The reconstruction quality from the PA measurements is compared in Figure 10 in terms of SNR. NLCS-est achieves significant better SNR than standard CS. Note that some of the SNR degradation is due to higher thermal noise at high efficiency levels, which impacts both CS and NLCS. We note that the SNR in an uncompressed setting resembles that of the CS results.

\section{CONCLUSION}

In this paper, we present a nonlinear compressive sensing formulation and associated optimization algorithms. We consider the scenario where compressive sensing measurements are distorted due to analog characteristics of sensing devices. We note that these distortion functions are near-isometry, and prove that information can be well-preserved despite these distortions. We then present optimization algorithms to solve 


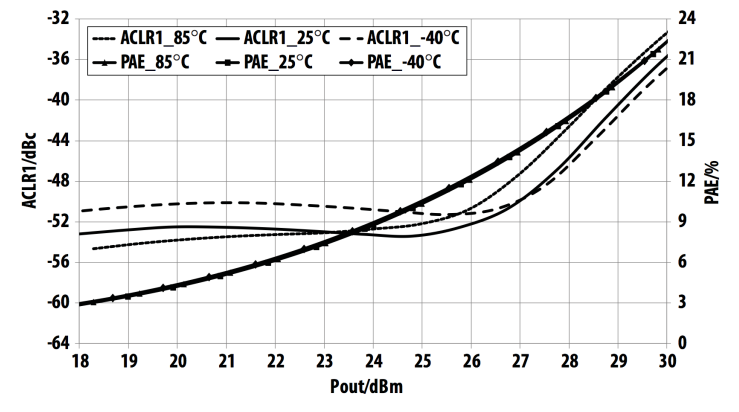

Fig. 9. Nonlinearity and power efficiency of the MGA-43003 power amplifier [15]. ACLR1 on the left $y$-axis represents the level of nonlinearity. Power-added efficiency (PAE) on the right $y$-axis represents power efficiency. Note that nonlinearity increases with higher power efficiency. When output power is below $26 \mathrm{dBm}$, the systems behaves linearly.

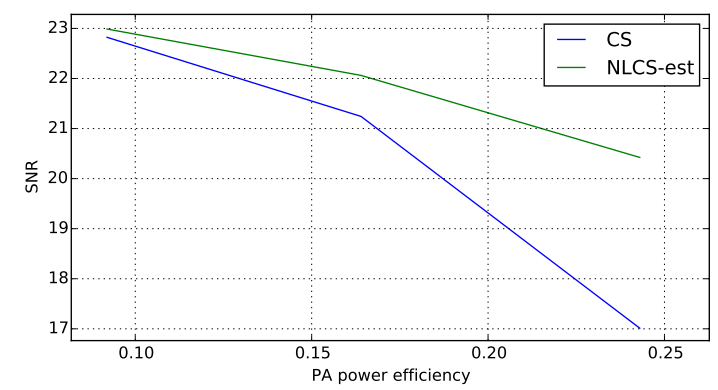

Fig. 10. Reconstruction error in $\ell_{2}$ norm of NLCS in comparison to standard compressive sensing using measurements from a real power amplifier. Operating the amplifier at higher efficiency levels causes distortion. As a reference, the first sample point around $9 \%$ efficiency is at the edge of the linear response region with output power around $23 \mathrm{dBm}$ (see Figure 9).

the NLCS problem, which can estimate the distortion function while performing signal recovery.

We validate the proposed methodology on both simulated and real world data. We demonstrate that modeling nonlinearity directly enables us to lower the linearity requirements for power amplifiers, which results in better power efficiency. Beyond the gains discussed in this paper, we believe the same principle can be applied to other devices with similar properties, as shown in the simulated results.

\section{ACKNOWLEDGEMENTS}

This work is supported in part by gifts from the Intel Corporation and in part by the Naval Supply Systems Command award under the Naval Postgraduate School Agreements No. N00244-15-0050 and No. N00244-16-1-0018.

\section{REFERENCES}

[1] D. L. Donoho, "Compressed sensing," Information Theory, IEEE Transactions on, vol. 52, no. 4, pp. 1289-1306, 2006.

[2] E. J. Candes and T. Tao, "Near-optimal signal recovery from random projections: Universal encoding strategies?" Information Theory, IEEE Transactions on, vol. 52, no. 12, pp. 5406-5425, 2006.

[3] J. C. Pedro, N. B. Carvalho, C. Fager, and J. A. Garcia, "Linearity versus efficiency in mobile handset power amplifiers: A battle without a loser," Microwave Engineering Europe (August 2004), 2004.
[4] L. Jacques, J. N. Laska, P. T. Boufounos, and R. G. Baraniuk, "Robust 1bit compressive sensing via binary stable embeddings of sparse vectors," IEEE Transactions on Information Theory, vol. 59, no. 4, pp. 20822102, 2013.

[5] P. T. Boufounos, "Reconstruction of sparse signals from distorted randomized measurements," in 2010 IEEE International Conference on Acoustics, Speech and Signal Processing. IEEE, 2010, pp. 3998-4001.

[6] T. Blumensath, "Compressed sensing with nonlinear observations and related nonlinear optimization problems," IEEE Transactions on Information Theory, vol. 59, no. 6, pp. 3466-3474, 2013.

[7] C. Thrampoulidis, E. Abbasi, and B. Hassibi, "Lasso with non-linear measurements is equivalent to one with linear measurements," in $\mathrm{Ad}$ vances in Neural Information Processing Systems, 2015, pp. 3420-3428.

[8] J. Vuolevi and T. Rahkonen, Distortion in RF power amplifiers. Artech house, 2003.

[9] I. Teikari, Digital predistortion linearization methods for RF power amplifiers. Helsinki University of Technology, 2008.

[10] D. Schreurs, M. O'Droma, A. A. Goacher, and M. Gadringer, $R F$ power amplifier behavioral modeling. Cambridge University Press New York, NY, USA, 2008.

[11] J. Liu, "Practical behavioral modeling technique of power amplifiers based on loadpull measurements," 2005.

[12] A. Beck and M. Teboulle, "A fast iterative shrinkage-thresholding algorithm for linear inverse problems," SIAM journal on imaging sciences, vol. 2, no. 1, pp. 183-202, 2009.

[13] B. Odonoghue and E. Candes, "Adaptive restart for accelerated gradient schemes," Foundations of computational mathematics, vol. 15 , no. 3 , pp. 715-732, 2015.

[14] Y. Nesterov, "Efficiency of coordinate descent methods on huge-scale optimization problems," SIAM Journal on Optimization, vol. 22, no. 2, pp. 341-362, 2012.

[15] A. Technologies. (2016) Mga-43003. [Online]. Available: http: //www.avagotech.com/docs/AV02-4350EN 